\title{
The best joint pharmacy and diabetes team initiative to improve insulin and prescribing safety in hospital
}

\author{
UMESH DASHORA, ${ }^{1}$ MIKE SAMPSON, ${ }^{2}$ ERWIN CASTRO, ${ }^{1}$ DEBBIE STANISSTREET, ${ }^{3}$ CHRISTINE JONES, ${ }^{2}$ \\ ROWAN HILLSON ${ }^{4}$ ON BEHALF OF JOINT BRITISH DIABETES SOCIETIES FOR INPATIENT CARE
}

\begin{abstract}
Introduction: The annual National Diabetes Inpatient Audit (NaDIA) in the UK continues to show a high incidence of insulin errors in patients admitted to hospital with diabetes. It is clear that new initiatives are urgently required to address this risk.

Methods: The Joint British Diabetes Societies for In-Patient Care (JBDS-IP) organised the third national Rowan Hillson Insulin Safety Award on the theme of the best joint pharmacy and diabetes team initiative to improve insulin and prescribing safety in hospital.

Results: There were two winners (Derby and Sheffield teams) and one runner up (St George's Hospital team). The Derby team won by showing improvements in NaDIA-reported data on medication, prescription, glucose management and insulin errors by a combination of electronic prescribing, staff education and regular audits. The Sheffield team improved self-administration of insulin by a pharmacist-led programme. The St George's Hospital team improved patient safety by a dedicated pharmacist-supported programme for peri-operative patients.

Conclusions: These and similar schemes need to be developed, promoted and shared to reduce insulin errors in hospitalised patients with diabetes.

Br J Diabetes 2018;18:163-166
\end{abstract}

Key words: insulin safety, award, pharmacy, initiative

Diabetes and Endocrine Centre, Conquest Hospital, Hastings, UK

Elsie Bertram Diabetes Centre, Norfolk and Norwich University Hospitals NHS Foundation Trust, Norwich, UK

3 Diabetes Centre, Lister Hospital, East and North Hertfordshire NHS Trust, UK

4 Former National Clinical Director for Diabetes, Department of Health, UK

Address for correspondence: Dr Umesh Dashora

Consultant, Diabetes and Endocrinology, Conquest Hospital, The Ridge,

St Leonards on Sea, East Sussex TN7 7RD, UK

Tel: +44 (0)1424 755255

E-mail: u.dashora@nhs.net

https://doi.org/10.15277/bjd.2018.195

\section{Results of Rowan Hillson Insulin Safety Award}

Joint Winners:

Dr Suma Sugunendran suma.sugunendran@nhs.net, Consultant Diabetologist and team, Royal Derby Hospitals for 'Derby Diabetes Inpatient Improvement Projects'

Sallianne Kavanagh, sallianne.kavanagh@sth.nhs.uk, Lead Clinical Pharmacist and team, Sheffield Teaching Hospital for

'A pharmacist led initiative to support insulin administration'

Runner up:

Sara Qureshi, Sara.Qureshi@stgeorges.nhs.uk, Lead Pharmacist, Theatres and team, St George's University Hospital for 'Operation Pharmacist: AQ:Ending the highs and lows of diabetes'

\section{Introduction}

Insulin and medication errors are common and preventable. Since the first National Diabetes Inpatient Audit (NaDIA), a number of attempts such as bespoke e-learning modules, "Think Glucose" programmes, education of junior doctors regarding diabetes management during their induction and modifications in prescription charts have been developed to improve insulin safety. As a result, between 2010 and 2017 subsequent NaDIA results demonstrated some reduction in errors related to inappropriate insulin infusions (7.4\% to $6.3 \%$ ), medication errors ( $44.5 \%$ to $31.3 \%$ ), prescribing errors $(30.7 \%$ to $19.0 \%)$, glucose management errors ( $24.1 \%$ to $18.5 \%$ ), insulin errors ( $25.8 \%$ to $18.6 \%$ ), reduction in mild hypoglycaemia (22.8\% to $16.7 \%$ ) and severe hypoglycaemia ( $11.8 \%$ to $7.1 \%$ ) in hospitalised patients with diabetes in the UK. ${ }^{1}$ The NaDIA results also showed that there is considerable variation between trusts - and sometimes at different times within the same trust. ${ }^{1}$ Clearly, further improvements are needed to improve patient safety in hospitalised people with diabetes.

Following the success of previous contests to find 'the best insulin prescription chart'2 and 'the best hypoglycaemia avoidance initiative', ${ }^{3}$ the Joint British Diabetes Societies for In-Patient Care (JBDS-IP) launched a national competition in 2016 to find joint pharmacy and diabetes team innovations with positive impact on insulin safety so that the best and effective practices can be identified, rewarded and shared with the other trusts in the UK.

Rowan Hillson chaired the judging panel, which consisted of Dr 
Daniel Flanagan, Consultant Diabetologist, Plymouth Hospitals NHS Trust; Dr Clare Crowley, Medicines Safety Pharmacist, Oxford University Hospital NHS Trust; Nicola Wake, Lead Clinical Pharmacist, Northumberland Healthcare NHS Foundation Trust; Gillian Cavell, Consultant Pharmacist, King's College Hospital NHS Foundation Trust; and Caroline Brooks, Diabetes Inpatient Specialist Nurse, Maidstone and Tunbridge Wells NHS Foundation Trust.

\section{Results}

There were 19 very high quality entries for the awards. After painstaking evaluation and assessment as per pre-defined criteria, the winning entries were selected. The judges were particularly impressed when high quality data was included with the project and there was evidence of clear improvement with the initiative. Adaptability of the project was also scored highly. Dr Suma Sugunendran, Consultant Diabetologist from Royal Derby Hospital NHS Foundation Trust, and Sallianne Kavanagh, Lead Clinical Pharmacist from Sheffield Teaching Hospital NHS Foundation Trust, were judged as the joint winners of the competition and Sara Qureshi, Lead Pharmacist, Theatre and Anaesthetics from St George's University Hospital NHS Foundation Trust, secured the runner-up position.

\section{Winning entry: Derby Insulin Safety Group}

The winning team from Derby created a Derby Insulin Safety Group (ISG) comprising a lead diabetes specialist nurse, senior pharmacist, head of in-patient safety, quality and improvement and consultant diabetologist. They met monthly to analyse incident reports, any significant or never events and objective data from point of care testing, with the overall aim of improving key NaDIA outcomes. A package of projects was designed with the aim of improving in-patient diabetes care including:

- Modification of electronic prescriptions to minimise risks

- Rolling out of staff education through different methods

- Targeted education of specific wards

- Inclusion of key diabetes parameters in the monthly ward assurance audit performed by senior nurses

\section{Mini project 1: Optimisation of electronic prescription and medicines administration (EPMA)}

Collaborating with pharmacists and IT, an electronic prescription chart was created with features such as drop-down options, especially for short acting and biphasic insulins restricted to meal times, prompts for the need of a meal with insulin, triggers to prevent confusion of insulin concentration (ie, $100 \mathrm{u} / \mathrm{mL}$ with dose), alerts to avoid stat doses of rapid acting insulin and requirement of capillary blood glucose (CBG) entry at the time of insulin administration. Active guidance and support from the pharmacist was crucial in achieving these changes to the electronic prescription.

\section{Mini project 2: Increased staff awareness and education}

This project involved creating a web of link nurses throughout all the wards, mandatory training for safe use of insulin, junior doctors training in safe use of insulin, credit card size information package for insulin profiles, flow chart for management of acute hyperglycaemia and hypoglycaemia, insulin profiles charts on all drug trolleys and drug rooms and targeted education to specific wards and healthcare professionals (HCPs) as needed.

\section{Mini project 3: Regular quality assurance}

The third mini project involved senior ward nurses regularly auditing appropriate management of acute hyperglycaemia and acute hypoglycaemia in their wards and screening of all patients with diabetes. Regular meetings were conducted to review the progress of all the projects and all the errors identified by Datix and hypoglycaemia reports.

The momentum of the entire package of projects was driven by regular meetings with review of all the errors (Datix and hypos) and the progress of the projects. Incident reports reduced from 175 in 2013-14 to 133 in 2015-16. Between 2013 and 2015, NaDIA results showed a reduction in errors related to medications $(41.4 \%$ to $27.1 \%)$, prescriptions (14.9\% to $8.3 \%)$, glucose management (29.9\% to $19.8 \%$ ) and insulin (20.7\% to $10.4 \%)$. Interestingly, the corresponding national mean figures for these errors actually increased slightly during this period. Foot assessments improved from $17.1 \%$ to $50 \%$ and severe hypos reduced from $9.8 \%$ to $4.4 \%$; the national figures remained unchanged during this period. The ISG was voted by hospital staff as winner of the Trust's 'celebrating success award' and was asked to share the innovation at the Diabetes UK conference. The improvements were achieved at a very low cost, but may require some investment for these to be sustained. Involvement of the head of in-patient safety quality made it easier to implement the project across various parts of the organisation.

\section{Joint winner: Sheffield pharmacy-led project for self-administration of insulin}

A retrospective review of the incident reporting system (Datix) for 1 year showed that there were a number of incidents in relation to insulin administration to patients including omission (20\%), delayed $(0 \%)$, wrongly prescribed $(4 \%)$, preventable incidents if patients had been self-administering (40\%), duplicate (4\%), wrong doses/insulin (8\%) and wrong patients (0\%) amounting to 51 Datix reports of in-patient insulin incidents which included intravenous incidents. It was thought that $40 \%$ of incidents could have been prevented if the patient had been self-administering whereas 12\% (6) incidents occurred in patients who were self-injecting.

A patient focus group identified some of the concerns patients had when coming to hospital, including not having access to their insulin and doses not being given at the correct time in relation to meals with resulting hypoglycaemia. The team presented self-administration as a solution and appraised all the options (Table 1).

In the end, however, the cost of any of these options to be provided for individual and all patients was prohibitive. The final agreement was to give possession of insulin to patients without a formal assessment of competence but under clinical governance of a pharmacist. The pharmacist reviewed the patients, updated the selfadministration status on the drug chart, prescribed basal insulin and requested blood glucose and ketone testing if needed.

A re-audit showed a significant number of patients (50\%) were self-administering insulin, some with supervision and help from 
Table 1 Appraisal of all the options of insulin storage to facilitate self-administration

Storage option

\begin{tabular}{|c|c|c|c|c|c|c|c|c|c|c|c|c|c|}
\hline & \multicolumn{2}{|c|}{$\begin{array}{c}\text { Pharmacy } \\
\text { service } \\
\text { compatible }\end{array}$} & \multicolumn{4}{|c|}{ Security } & \multirow{2}{*}{ 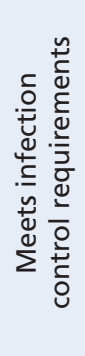 } & \multicolumn{3}{|c|}{$\begin{array}{c}\text { Nurse } \\
\text { accessibility }\end{array}$} & \multicolumn{3}{|c|}{$\begin{array}{c}\text { Patient } \\
\text { accessibility }\end{array}$} \\
\hline & $\begin{array}{l}0 \\
\frac{0}{0} \\
3 \\
0 \\
0 \\
0\end{array}$ & 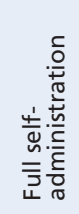 & 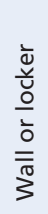 & 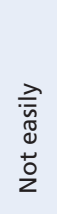 & 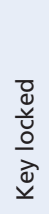 & 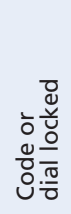 & & 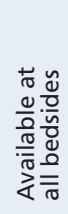 & 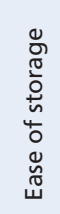 & 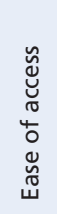 & 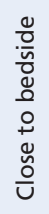 & 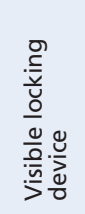 & 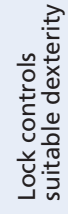 \\
\hline Utilisation of current locker & $x$ & $\sqrt{ }$ & $\sqrt{ }$ & $\sqrt{ }$ & $\sqrt{ }$ & $x$ & $\sqrt{ }$ & $\sqrt{ }$ & $\sqrt{ }$ & $\sqrt{ }$ & $\sqrt{ }$ & $\sqrt{ }$ & $\sqrt{ }$ \\
\hline Additional wall locker & $\sqrt{ }$ & $\sqrt{ }$ & $\sqrt{ }$ & $\sqrt{ }$ & $\sqrt{ }$ & $x$ & $\sqrt{ }$ & $\sqrt{ }$ & $\sqrt{ }$ & $\sqrt{ }$ & $\sqrt{ }$ & $\sqrt{ }$ & $\sqrt{ }$ \\
\hline Additional bedside cabinet & $\sqrt{ }$ & $\sqrt{ }$ & $\sqrt{ }$ & $\sqrt{ } 1$ & $\sqrt{ }$ & $x$ & $\sqrt{ }$ & $x$ & $x$ & $x$ & $\sqrt{ }$ & $\sqrt{ }$ & $\sqrt{ }$ \\
\hline Large cashier box & $\sqrt{ }$ & $\sqrt{ }$ & $x$ & $x$ & $\sqrt{ }$ & $x$ & $\sqrt{ }$ & $x$ & $\sqrt{ }$ & $\sqrt{ }$ & $\sqrt{ }$ & $\sqrt{ }$ & $\sqrt{ }$ \\
\hline Small cashier box & $\sqrt{ }$ & $\sqrt{ }$ & $x$ & $x$ & $\sqrt{ }$ & $x$ & $\sqrt{ }$ & $x$ & $\sqrt{ }$ & $\sqrt{ }$ & $\sqrt{ }$ & $\sqrt{ }$ & $\sqrt{ }$ \\
\hline Locked bags & $\sqrt{ }$ & $\sqrt{ }$ & $x$ & $x$ & $\sqrt{ }$ & $x$ & $x$ & $x$ & $\sqrt{ }$ & $\sqrt{ }$ & $\sqrt{ }$ & $\sqrt{ }$ & $\sqrt{ }$ \\
\hline Patient responsibility & $\sqrt{ }$ & $\sqrt{ }$ & $x$ & $x$ & $x$ & $\mathrm{x}$ & $\sqrt{ }$ & $\sqrt{ }$ & $\sqrt{ }$ & $\sqrt{ }$ & $\sqrt{ }$ & $\mathrm{x}$ & $x$ \\
\hline Ward trolley & $x$ & $\mathrm{x}$ & $x$ & $\sqrt{ }$ & $\sqrt{ }$ & $x$ & $\sqrt{ }$ & $x$ & $x$ & $\sqrt{ }$ & $x$ & $\sqrt{ }$ & $x$ \\
\hline Medical metal storage box & $\sqrt{ }$ & $\sqrt{ }$ & $x$ & $\sqrt{2}$ & $\sqrt{ }$ & $x$ & $\sqrt{ }$ & $x$ & $\sqrt{ }$ & $\sqrt{ }$ & $\sqrt{ }$ & $\sqrt{ }$ & $\sqrt{ }$ \\
\hline Drug return box & $\sqrt{ }$ & $\sqrt{ }$ & $\sqrt{ }$ & $\sqrt{ }$ & $\sqrt{ }$ & $\sqrt{ }$ & $\sqrt{ }$ & $\sqrt{ }$ & $\sqrt{ }$ & $\sqrt{ }$ & $\sqrt{ }$ & $\sqrt{ }$ & $\sqrt{ }$ \\
\hline Plastic medication wall box & $\sqrt{ }$ & $\sqrt{ }$ & $\sqrt{ }$ & $\sqrt{ }$ & $\sqrt{ }$ & $\sqrt{ }$ & $\sqrt{ }$ & $\sqrt{ }$ & $\sqrt{ }$ & $\sqrt{ }$ & $\sqrt{ }$ & $\sqrt{ }$ & $\sqrt{ }$ \\
\hline
\end{tabular}

nurses. The administrative burden on nurses was reduced while the patients felt empowered. It was felt that to continue pharmacist- supported self-administration of insulin would require one whole-time equivalent pharmacist for both sites of the Trust. The main challenge was to overcome the pre-conceptions of the governance teams about the potential risk of a patient or visitor misappropriating insulin and causing harm to self or others. Feedback from the staff and patients was very positive and they valued this service.

\section{Runner-up entry: High and low glucose levels}

In this initiative, diabetes specialist prescribing pharmacists optimised medicines management in the pre-operative setting at St George's Hospital, agreed a written proactive glycaemic management plan involving patients and identified and escalated any complex issues to the diabetes team resulting in seamless collaboration between teams, reduction in last minute cancellations, better glycaemic control from the pre-operative stage through theatres, recovery, ward and finally discharge and excellent feedback from surgeons, anaesthetists, the diabetes team and patients. Unnecessary variable rate intravenous insulin infusions (VRIII) were reduced, length

\section{Product characteristics}

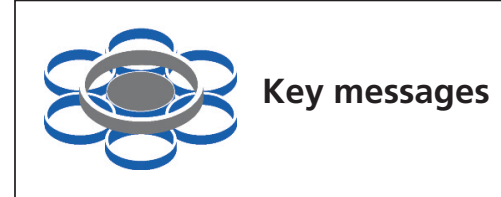

- The Rowan Hillson Insulin Safety Award is an initiative developed to identify best practices in the UK to improve insulin safety

- The 2016 round was to find the best joint pharmacy and diabetes team initiative to improve insulin and prescribing safety in hospital

- There were two joint winners:

(1) The Derby team submitted their project which essentially consisted of electronic prescribing developed with support from the pharmacists, staff education targeted for specific areas and a monthly ward assurance audit performed by senior nurses.

(2) The Sheffield team described a pharmacist-led project of supporting self-administration of insulin by leaving the insulin with patients and supporting them to self-administer

- The runner-up team from St George's Hospital entered a pharmacist-led initiative to support patients in the peri-operative setting which led to a significant reduction in last minute cancellations of surgery and a positive experience of healthcare professionals and patients 


\section{GLUCOSE MONITORING}

THAT FITS INTO YOURPATIENTS DA Y LIF' 2.2 . 政 (1) (1)

\section{Digital health tools that work together} for seamless diabetes management

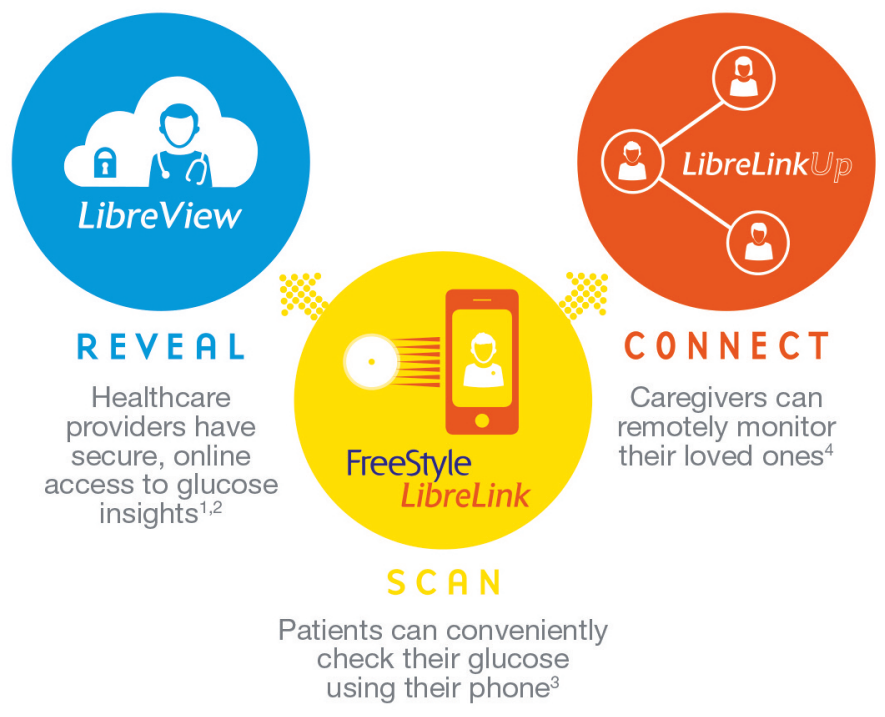

Find out more at www.FreeStyleDiabetes.co.uk

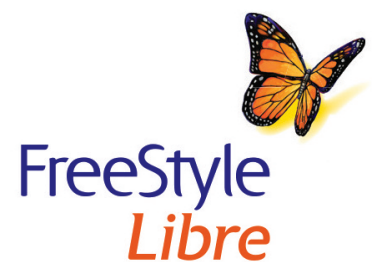

FLASH GLUCOSE MONITORING SYSTEM

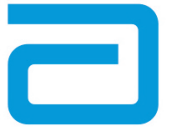

Abbott

1. LibreView data can be viewed in the Safari Browser on Mac OS X Mountain Lion or higher computers and on iOS 6 or higher mobile devices. Currently, uploading of glucose data is only supported on Windows-based computers. Minimum system requirements are Windows Vista with III. 512MB DRAM, 2G Hard Drive, USB 2.0, LCD Screen with resolution of 1024x768. 2. LibreView (including the Libre LinkUp mobile app) is not intended to be a primary glucose monitor: home users must consult their primary device(s) and consult a healthcare professional before making any medical interpretation and therapy adjustments from the information in the software, and healthcare professionals should use information in the software in conjunction with other clinical information available to them. 3 . The FreeStyle LibreLink app is compatible with NFC enabled phones running Android OS 5.0 or higher and with iPhone 7 and higher running OS 11 and higher. 4. LibreLinkUp is a mobile application, developed and provided by Newyu, Inc. Use of LibreLinkUp requires registration with LibreView, a service provided by Abbott and Newyu, Inc.

FreeStyle, Libre and related brand marks are trademarks of Abbott Diabetes Care, Inc. In various jurisdictions. Date of preparation: May 2018. ADCMDP180062

Continued from page 165

of stay reduced, day admissions increased, night before admissions reduced, appropriate VRIII increased and timely diabetologist input secured. The initiative received various awards including the Pharmaceutical Care award 2015, the Trust's clinical audit award and Quality in Care diabetes award.

The initiative led to the avoidance of an estimated 10 cancellations of surgeries (saving $\mathrm{f15,000)}$ and a reduction of 105

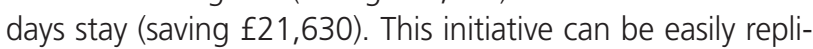
cated with some investment and support in prescribing courses for specialist pharmacists and it can reduce pressure on doctors and specialist nurses. The initiative was able to ensure all patients' admission glucose levels were between 4 and $12 \mathrm{mmol} / \mathrm{L}$. Maintaining CBG between 4 and $12 \mathrm{mmol} / \mathrm{L}$ throughout the patients' journey was a challenge (52.4\%) and would require ongoing support and education from the diabetes team.

\section{Summary and conclusions}

In summary, the competition revealed some excellent joint practices between diabetes and pharmacy teams that can be utilised and adapted by other trusts to improve insulin safety and management of in-patients with diabetes. Two initiatives were judged to be joint winners. The Derby initiative used electronic prescribing in conjunction with targeted education and audits whereas the Sheffield project implemented a support system to facilitate self-administration of insulin. The St George's Hospital innovation was runner-up, where a prescribing pharmacist regularly supported patients with diabetes in the pre-operative setting.

Acknowledgements: The Rowan Hillson Insulin Safety Award is run by the Joint British Diabetes Societies In-Patient (JBDS-IP) group with financial support from the Association of British Clinical Diabetologists (ABCD) and Diabetes UK.

\section{Conflict of interest: None \\ Funding: None}

\section{References}

1. NHS Digital. National Diabetes Inpatient Audit 2017. https://digital.nhs.uk/data-and-information/publications/statistical/nationaldiabetes-inpatient-audit/national-diabetes-inpatient-audit-nadia-2 017 (accessed 29 April 2018)

2 Dashora U, Sampson MJ, Castro E, et al. Rowan Hillson Insulin Safety Award 'best in class' insulin prescription chart competition. Br J Diabetes 2015;15:135-8.

3 Dashora U, Sampson MJ, Castro E, et al. The best hypoglycaemia avoidance initiative in the UK. Br J Diabetes 2017;17:74-7. 